\title{
ALGUNAS CUESTIONES EMERGENTES EN EL DOMINIO DE LA CIENCIA DE LA INFORMACIÓN'.
}

\author{
ALGUMAS QUESTÕES EMERGENTES NO DOMÍNIO DA \\ CIÊNCIA DA INFORMAÇÃO
}

José López Yepes *

\begin{abstract}
RESUMEN
Introducción: el presente artículo hace una reflexión sobre los temas emergentes en el área de la Ciencia de la Información $(\mathrm{Cl})$. Presenta algunos conceptos en evolución relacionados al área y discute aspectos en torno a las pesquisas que ya están andando, así como evaluar el papel de los profesionales de la información en el ámbito de la investigación científica. Objetivo: Discutir sobre lo que es la $\mathrm{Cl}$. Entender como están siendo evaluadas las pesquisas en este campo científico. Estudiar cómo son vistos actualmente los profesionales de la información cuáles son las competencias deseadas para estos. Metodología: Pesquisa bibliográfica. Resultados: Los resultados indican que la $\mathrm{Cl}$ es una ciencia social, informativa/comunicativa que tiene como objeto de estudio un proceso informacional que consiste en retener, recuperar y transformar la información para la generación de nuevos conocimientos. En lo que se refiere a las investigaciones científicas, la $\mathrm{Cl}$ agrupa diversas áreas de la ciencia que tienen como objeto de estudio el proceso informativo documental, siendo la mayor parte de las pesquisas, de naturaleza cualitativa. Dentro de las diversas competencias del profesional de la información el estudio enfatiza el ejercicio de aprendizaje de lectura, una vez que dicho profesional actúa directamente en diferentes áreas del conocimiento. Conclusiones: el estudio se enfocó en el área de la $\mathrm{Cl}$ en tres aspectos, siendo ellos: lo que es la $\mathrm{Cl}$, cuáles son las pesquisas más desarrolladas en el campo y cuáles son las habilidades de los profesionales de la información. La Cl es un área en crecimiento y necesita de un debate permanente, principalmente investigaciones que abarquen esos tres aspectos mencionados, una vez que los mismos se complementan.

Palabras clave: Ciencia de la información. Proceso Informacional. Profesional de la Información. Competencias del profesional de la información.
\end{abstract}

\footnotetext{
${ }^{1}$ Conferencia inaugural pronunciada en el VI Seminario de Ciencia de la Información. Londrina, Universidad Estadual de Londrina, 3-6 de agosto de 2016.

*Doctor en Filosofía y Letras. Facultad de Ciencias de la Información, Universidad Complutense de Madrid. E-mail: yepes@ucm.es.
}

Inf. Inf., Londrina, v. 22, n. 2, p. 320 - 339, maio/ago., 2017. http:www.uel.br/revistas/informacao/ 


\section{INTRODUÇÃO}

Un excelso poeta en lengua portuguesa, Luis de Camoens, que también escribía en español, decía en la segunda octava de su obra Os Lusiadas publicada en Lisboa en 1572: Cantando espelharei por toda parte/ se a tanto me ayudar o engenho e arte. Quisiera yo mismo buscar servirme del arte y del ingenio para servirme de dos características de la ficción literaria, a saber: sentir una nueva experiencia positiva en este escenario y hallar la certidumbre de mis ideas. Pero también quisiera regresar al lugar de donde vengo enriquecido por las dudas, observaciones y sugerencias que se me ofrezcan.

Me encuentro ahora en un foro privilegiado para "apresentação e para discussão da pesquisa científica em Ciência da Informação, congregando pesquisadores, profissionais e estudantes, visando promover a discussão de temáticas de interesse da área". Pues bien, yo quiero sumarme modestamente a este espacio de debate y de cuestiones controvertidas con la esperanza de obtener ideas útiles. En efecto, debemos hablar de fenómenos emergentes pues parece que lo emergente es consustancial a nuestro campo de estudio, sin duda, fértil campo de innovaciones terminológicas y conceptuales. Todos los días inventamos nuevas palabras para, con cierta frecuencia, decir lo mismo o bautizar un concepto que tal vez no merezca haber visto la luz. Llamamos fenómeno a una "cosa extraordinaria y sorprendente". Y emerger es brotar -salir a la superficie- algo nuevo y tal vez inesperado. Desde mi punto de vista, y a la hora de seleccionar algunas cuestiones de tal naturaleza, he tenido en cuenta un doble criterio: considerar que emergente no sólo es el pensamiento que brota inesperadamente sino también el que sigue brotando por que no acabamos de canalizarlo y convertirlo en líquido fecundo y provechoso. Se trata, pues, de cuestiones que vertebran nuestro campo y que siguen produciendo literatura sin cesar. ¿Y cual es la razón? : Porque no están del todo resueltas. Me voy a referir, por consiguiente y sucesivamente, a algunos conceptos y denominaciones de nuestro campo académico y sus 
aplicaciones; a los problemas derivados de la investigación y sus tendencias y a los que atañen a la definición del profesional de la información y a su formación investigadora. El segundo criterio de selección se basa en el hecho de que dichas cuestiones hayan sido objeto de reflexión en alguna de mis publicaciones o de que mi modesta experiencia pueda ayudar a vislumbrarlas con cierta claridad. En fin, sobre la base de ambos criterios me propongo responder y reflexionar, según mi entender, acerca de tres cuestiones: ¿Qué es la Ciencia de la Información? ¿Cómo evaluar la calidad de la investigación en nuestro campo? y ¿Qué es un bibliotecario, documentalista o profesional de la información? y ¿Cuáles son las competencias vinculadas a su formación investigadora. Ciertamente cuestiones de permanente debate.

\section{PRIMERA CUESTIÓN. EL CONCEPTO DE LA DISCIPLINA: ¿QUÉ ES LA CIENCIA DE LA INFORMACIÓN?}

Resulta llamativo que los juristas, historiadores, médicos, químicos, etc. no suelen dudar acerca de la naturaleza y los objetivos de sus respectivas disciplinas. Sin embargo, el estudio de la naturaleza de nuestra disciplina es un tema presente en numerosos eventos. Lo que provoca que, a luz del llamado cambio documentario, se convierte con mucha frecuencia en una expedición a la búsqueda de un paradigma científico. Muchos son los que se afanan en la búsqueda del paradigma como si se tratara del oro ambicionado por los buscadores de Alaska o por los antiguos descubridores españoles y portugueses. Resulta paradójico pensar que, si hasta mediados del siglo XIX, la comunidad científica parecía tener claros los conceptos esenciales en torno a las disciplinas del documento- Archivística, Biblioteconomía, Bibliografía, Museología- aunque aparecieran separadas. Sin embargo, la eclosión de la ciencia moderna con el incesante aumento de la bibliografía, las crecientes necesidades de información y la revolución tecnológica determinaron nuevas concepciones a partir de las teorías gestadas en Norteamérica y en Europa. En este escenario hay un denominador común: la Información, palabra mágica. Sin duda, el término información posee contornos harto ambiguos, de universal 
consideración y, en consecuencia, de dificultosa definición. Pero, ¿qué es la información? La información es -como indica su etimología- la acción de dar forma a algo. Por extensión podemos afirmar que se trata de dar forma a un mensaje de un modo determinado para su transmisión a través de un medio determinado. Dos nociones esenciales: modo y medio. Ello permite considerar la información como un modo de adecuación de un mensaje transmisible a través de un medio de difusión individual o colectiva. Pues bien, son modos informativos y disciplinas consecuentes el periodismo, la comunicación audiovisual, la publicidad, la propaganda, las relaciones públicas, la documentación... y son medios informativos la voz, la prensa, la radio, la televisión, la valla publicitaria, Internet, etc.

Desde esta perspectiva, hemos conformado una denominada teoría comunicativa de la Ciencia de la Información cuyos elementos paradigmáticos serían los siguientes:

a) La Ciencia de la Información es una ciencia para la ciencia, por su carácter instrumental al servicio del crecimiento, difusión y evaluación de todos los saberes.

b) Es una ciencia de la comunicación documentaria ya que incluye un proceso informativo con sus típicos elemento: sujetos emisores, mensaje, medio y sujetos receptores.

c) Es una ciencia integradora de todas las disciplinas del documento lo que permite aceptar la expresión Ciencias de la Información como ámbito que incluye la Archivística y la Museología.

d) Tal denominación no define qué tipo de información manejamos por lo que, en mi opinión, deberíamos decir Ciencia o Ciencias de la Información documental.

En suma, Biblioteconomía/Documentación/Ciencia de la Información se ocupa de un modo de informar a través de diversos medios informativos lo que comporta que, en su desempeño, tenga lugar un proceso informativodocumental en el que se produce información documental. $\mathrm{Y}$, a mayor 
abundamiento, podríamos completar esta noción afirmando que nuestro campo es una ciencia social, informativo-comunicativa que tiene como objeto de estudio un proceso informativo consistente en la retención, recuperación y transformación de mensajes producidos en procesos informativos anteriores y cuyos mensajes se comunican transformados como fuentes de información para obtener nuevo conocimiento o para la acertada toma de decisiones. La nueva información producida es información documental o fuente actual de información a partir de la potencialidad consustancial a los mensajes conservados en cualquier lugar o tiempo. Si la información contingente es información de lo que pasa, la información documental es información de lo que queda (LÓPEZ YEPES, 2015).

\section{SEGUNDA CUESTIÓN: EL IMPACTO DE LA INVESTIGACIÓN Y LA EVALUACIÓN DE SU CALIDAD}

Como se sabe, la actual coyuntura tecnológica facilita grandemente la publicación o difusión de todo tipo de mensajes bien a través de los medios convencionales, bien a través de los medios que presta Internet. El profesor Codina ha escrito una frase ciertamente paradójica: "Cada vez tenemos más información, como consecuencia de los cual, la información cada vez tiene menos valor y, al mismo tiempo, cada vez tenemos acceso a más y más información gratuita y de más calidad" (CODINA BONILLA, 2015, p.7). Lo cierto es que la información nos abruma en todas sus formas. Y el reto, cada vez más acuciante, es el de determinar su calidad, inversamente proporcional a la cantidad. Se requieren, pues, métodos que discriminen positivamente ambos parámetros. Si las redes, blogs, etc. nos facilitan señales de alerta, siguen siendo las revistas los medios habituales de difusión y de evaluación de autores y trabajos.

La evaluación de la ciencia comprende diversos apartados, siendo algunos de los más notables los que siguen: a) Evaluación de la calidad de las revistas propiamente dichas mediante su factor de impacto; b) Evaluación de la calidad de los artículos mediante lista de los más citados; c) Evaluación de los 
autores mediante lista de los más citados; e) Evaluación de las instituciones que editan las revistas con mayor factor de impacto. Estas evaluaciones se llevan a cabo mediante variados criterios de orden cuantitativo y los resultados aparecen en bases de datos que aplican dichos criterios de modo cuantitativo bien en cuanto a la calidad editorial (LATINDEX, RESH, DICE, etc) o bien en cuanto a la calidad de contenidos mediante el número de citas recibidas por las revistas, los artículos, los autores y las instituciones (IN-RECS, RESH, JCR, REDALYC, etc.). La diversidad de las bases de datos evaluativas mediante recuento de citas y la renovada invención de indicadores bibliométricos ya nos anuncian la falta de criterios unánimemente aceptados y ciertos síntomas, por tanto, de inseguridad evaluativa.

Sin embargo, el problema de la evaluación no es inocuo puesto que de acertar en la misma depende el prestigio de los investigadores y determinadas recompensas no solo económicas sino también de promoción académica y profesional. Tal vez -y lamentablemente- estamos en un período de la historia de la ciencia en que la investigación ha dejado de ser una actividad de otium la simple búsqueda de la verdad en la terminología clásica- para pasar a ser un nec-otium, es decir, algo que se persigue con fines lucrativos en el sentido de persuadir a las agencias de evaluación de la calidad de nuestra investigación por medio de trabajos en los que, con frecuencia, junto a la presunta originalidad se advierte cierta escasa utilidad.

$Y$ ahora, inesperadamente, tras los indicadores bibliométricos, habitualmente utilizados para definir la calidad de autores, obras e instituciones, aparecen los indicadores alternativos o altmétricos. Sin duda, constituyen un concepto emergente que, como era de esperar, ya va produciendo ingente literatura. El nuevo enfoque se basa en la afirmación de que estos nuevos indicadores producidos en la web social proyectan información sobre el impacto de la ciencia más allá de los índice de citas. Son, pues, alternativa al tradicional uso de la medición del impacto de los trabajos y autores desde el número de citas recibidas. Estas altmétricas han sido definidas como una "familia de indicadores que miden e impacto de la investigación cuantificando su presencia en la web social: número de tweets, 
menciones en blogs, inclusión en marcadores sociales, presencia en gestores bibliográficos, etc." (BORREGO, 2014, p.4). Es cierto que vienen a contrarrestar algunas de las críticas que han recibido los métodos tradicionales e análisis de citas pero la utilidad de los nuevo indicadores, sin embargo, va a depender de su correcta interpretación y de la correcta recogida de datos. (Idem). Hoy día, los indicadores alternativos aparecen en dos grupos de fuentes: Bibliotecas y gestores de referencias digitales y Redes y medios sociales. En el primer sector tenemos como ejemplos el número de veces que algo ha sido favorito, de lectores o de grupos a los que se ha añadido. En el segundo sector se cuentan el número de menciones en redes sociales, en blosg, enciclopedias, etc. (TORRES-SALINAS; CABEZAS-CLAVIJO; JIMÉNEZCONTRERAS, 2013) como Google Scholar profiles, Linkedin, Mendeley, Slideshare y Twiter. En un trabajo de Torres Salinas y Milanés (2014) se analizan los indicadores alternativos para los autores mas productivos de la revista El Profesional de la Información y también la correlación entre ambos tipos de indicadores. Son agentes involucrados en estos procesos lo siguientes: proveedores de información, editores de revistas científicas y entidades que miden el impacto de la investigación con tales indicadores; repositorios, investigadores y los bibliotecarios Observamos el dominio, una vez más, de los cuantitativo, sin apenas resquicio para medir la calidad objetiva y la auténtica repercusión de los trabajos en el crecimiento de la ciencia. Concluimos este apartado afirmando que los indicadores altmétricos proporcionan visibilidad social gracias a la publicidad de autores y trabajos pero ello no comporta visibilidad académica o científica aunque, desde luego, puedan suponer un complemento para la actividad de difusión de la actividad científica entre los investigadores y en el medio social.

Todavía, los indicadores bibliométricos todavía se ven como la herramienta idónea para conocer la calidad de los contenidos y los de repercusión de las revistas científicas. En todo caso, calidad de contenidos y repercusión son factores que, con frecuencia, pueden solaparse. El consiguiente factor de impacto de revistas y artículos puede estar teñido de 
ruido si se trata de meras menciones indiscriminadas de los autores sin detectar claramente la relevancia de sus artículos.

Por ello, proponemos el uso de otra herramienta para la evaluación de la calidad de revistas, trabajos y autores. Es la que hemos nombrado análisis cualitativo de citas. Porque si el análisis cuantitativo de citas puede ser válido para reflejar la repercusión de algún modo de los elementos citados, no es necesariamente válido, en nuestra opinión, para conocer la verdadera repercusión en el crecimiento de la ciencia ya que se tienen en cuenta las citas de todo tipo de modo indiscriminado, por ejemplo, citas de agradecimiento, autocitas o autores en referencias bibliográficas que no han sido utilizados en los trabajos citantes, etc, es decir, citas no de calidad. A mayor abundamiento, la dudosa selección de las revistas-fuente motiva otro tipo de turbulencias que se reflejan en la falta de unanimidad en la evaluación ofrecida por las diversas bases de datos de evaluación cuantitativa, a lo que habría que añadir las dudas que algunos estudiosos han puesto de manifiesto en la actuación de los evaluadores de artículos.

El objetivo básico del análisis cualitativo de citas es, pues, evaluar la calidad de contenidos de revistas y artículos y la repercusión de las nuevas ideas contenidas en los mismos a lo largo del tiempo. Ello incluye la posibilidad de determinar:

1. El número de citas de calidad obtenidas por autores y trabajos.

2. El factor de impacto cualitativo de las revistas.

3. Las ideas de los demás utilizadas por cada autor (base intelectual deudora) y las ideas que de éste han tomado otros autores (base intelectual acreedora), lo que permite conocer el grado de madurez del investigador en el uso de las fuentes y su adscripción a determinadas escuelas o corrientes de pensamiento.

4. Las ideas propias obtenidas por cada autor así como su repercusión en el campo. 
5. El origen y evolución de líneas de investigación o contribución a la historia de una determinada disciplina y reconocimiento de las aportaciones de los respectivos autores.

Todo lo anterior puede contemplarse, por vía de ejemplo, en la base de datos cualitativa denominada Qualitas Scientiae, producto de una investigación financiada por el Ministerio español de Educación y Ciencia a nuestro grupo de investigación de la Complutense y en nuestros trabajos (LÓPEZ YEPES, 2003 ${ }^{\mathrm{a}}$ y b, y 2007), metodología de la que se han servido algunos autores de tesis doctorales asesoradas por miembros del grupo ${ }^{2}$.

Finalmente, no resisto la tentación de traer aquí la valoración que prestan los investigadores experimentados a sus lecturas y el método tradicionalmente utilizado para detectar el carácter científico de un texto. Basta con observar si se dan en él los siguientes parámetros: título expresivo y delimitativo del objeto de la investigación, qué problema trata de resolver y justificación de su necesidad, si emplea procedimientos metodológicos, redacción clara, uso de fuentes valiosas y presencia de conclusiones que signifiquen respuesta a los problemas planteados.

\section{TERCERA CUESTIÓN: EL PROFESIONAL DE LA INFORMACIÓN Y SU FORMACIÓN COMO INVESTIGADOR}

A los efectos de llegar a una actualización de la definición del profesional, les ruego que atiendan esta pequeña narración de uno de nuestros escritores de la llamada Generación del 98: "En la pequeña y vieja ciudad — escribe Azorín - hay dos, tres o cuatro hornos; la hornera tiene un marido o un hermano; este marido o este hermano es el anacalo. Se levanta el anacalo por la mañana, se desayuna y entre él y su mujer comienzan a llenar el horno de

\footnotetext{
2 Por ejemplo RIVERA, Martha Patricia. Análisis de la producción científica en revistas de biblioteconomía y documentación en México. Análisis del método cualitativo de citas. (20092013). Madrid, Facultad de Ciencias de la Documentación, Universidad Complutense de Madrid, 2015. Sobresaliente cum laude por unanimidad.
} 
leña y de hierbajos secos; luego lo encienden; un humillo azul surte por la chimenea y asciende ligeramente por el aire. El aire se llena de un grato olor de romero y de sabina quemados... Cuando el horno está ya encendido, sale el anacalo de casa... El anacalo recorre todas las casas del barrio; se asoma a la de Don Pedro y grita ¿Amasan?... Una voz grita desde dentro: No, y el anacalo se marcha... Nuestro amigo se halla ante la casa de Doña Asunción. La casa tiene un gran portalón con su puerta de roble pero esta puerta está siempre cerrada y a la casa se penetra por una estrecha puertecilla que existe en otra de las fachadas. El anacalo abre esta puertecilla y da un grito: ¿Amasan? Una voz replica: Sí... Recorre el anacalo varias dependencias y, al fin, se encuentra en el amasador; ésta es una estancia un poco sombría, se ven unas lejas llenas de perolitos, cazuelas, vasos; unos cedazos están colgados en la pared; en un ángulo, en una rinconera, reposa una orcita destinada a guardar la levadura; la artesa, grande y de pino, se halla colocada sobre dos travesaños empotrados en la pared, y encima de la artesa está el tablero lleno de panes blanco, recién amasados; un mandil rojo, verde, amarillo y azul los cubre, los abriga... $Y$ enseguida se pone una almohadilla redonda en la cabeza, coge el tablero, se lo coloca sobre el cráneo y se marcha. Éste es el oficio trascendental del anacalo: llevar el pan que va a ser cocido desde las casas al horno" (AZORÍN 1959, p. 81-83). El Diccionario de Autoridades de la RAE (1726-1739) definía anacalo como el criado o criada de la hornera que va a las casas particulares por el pan que se ha de cocer y lo trae al horno en unas tablas y, después de cocido, lo vuelve a llevar. Es voz muy usada en Toledo... dice ser arábiga y que viene del verbo necale que significa llevar de una parte a otra.

Creemos ver aquí la clave del problema en el tema de los contenidos, el hecho de poder controlar la capacidad ilimitada en nuestros días de fabricar contenidos científicos, empresariales y de opinión en forma de torrente indiscriminado y de dudoso valor mientras no se demuestre lo contrario. Las redes sociales y los blogs, por ejemplo, están erigiendo un imperio de opiniones que, por el mero hecho de incorporarlas al ciberespacio, pueden parecer ciertas y seguidas sin pestañear por numerosas personas. Creo que 
urge incorporar a los contenidos un valor añadido que asegure su aprovechamiento y conversión en auténtica y veraz fuente para la producción de nuevos contenidos. Dicho de otro modo: cocer el pan es añadir a la masa un nuevo valor. Somos anacalos que hacemos posible llevar la información a un escenario en el que, en sus contenidos, resplandezca la verdad del mensaje. De este modo, se facilita que la potencia informativa se transforme en acto, en noticia de actualidad, en nueva, verdadera y útil información.

Pienso honestamente que trasladar la información seleccionada y evaluada al usuario es nuestra tarea primordial, lo que implica el conocimiento lo más exhaustivo posible de esa información, el manejo eficaz de las herramientas de conserva y recuperación de la información y el desarrollo de técnicas de investigación capaces de desarrollar las nuevas ideas obtenidas en pro de la innovación en la sociedad. Desde estos presupuestos, postulamos que la misión del profesional de la información consista en sumar contenidos con valor añadido, es decir, contenidos científicos en su connotación de veraces y profundos y con el valor añadido de ser convertidos en fuente de nuevos contenidos.

A partir de aquí, pienso que, desde el enfoque de los contenidos, los profesionales de la información, deben poseer, además de las tradicionales, las siguientes competencias:

1. Expertos en elaboración de normas de asesoramiento y tutoría a fin de poder colaborar en la formación de nuevos investigadores y en sus equipos de investigación (LÓPEZ YEPES, 2015). El investigador no solo interpreta los mensajes de los documentos sino que los integra en su propio texto generando nuevos documentos y, por consiguiente, nuevos caminos de lectura crítica e interpretaciones. El corolario final de estas reflexiones puede conducirnos a desarrollar métodos para la formación del universitario en la lectura crítica.

2. Expertos en elaboración de normas para la evaluación de la ciencia: productividad de autores individuales o múltiples, evaluación de revistas, índices de citas, etc. 
3. Expertos en las tareas relativas a las aplicaciones de la web social en la investigación científica o comunidad de investigadores, con los que ha de mantener una eficaz relación en su triple vertiente de:

a) Compartir la investigación: redes sociales científicas, bases de datos de científicos, plataformas para la investigación y servicios instrumentales participativos.

b) Compartir los recursos: gestores de referencias bibliográficas, favoritos sociales e índices de citas.

c) Compartir los resultados: blogs y wikis, servicios de noticias científicas y acceso abierto.

¿Hacia dónde caminan las investigaciones sobre los modelos formativos? La inmensa mayoría de los autores habla de nuevos perfiles, de nuevas competencias y del mercado de trabajo. $Y$ aquí viene el tema de las famosas y reiteradas competencias. En este sentido observamos: 1) De un lado la tendencia a proponer competencias numerosas, tal vez excesivas y redundantes. 2) De otro, el carácter ambiguo del concepto de competencia que podría abarcar un conjunto de habilidades y destrezas que el egresado debe haber adquirido al concluir su ciclo formativo. En este sentido cabe hablar de competencias generales como las recogidas en el documento ATC215 (Assesment and Teaching of 21st Century Skills) promovido por la Universidad de Melbourne. Propone 10 competencias distribuidas en 4 grupos:

- Maneras de pensar: creatividad e innovación, pensamiento críticos, resolución de problemas, aprender a aprender.

- Herramientas para trabajar: Tecnologías digitales y manejo de la información.

- Maneras de trabajar: comunicación y colaboración

- Maneras de vivir en el mundo: fijación de metas, responsabilidad, papel en la comunidad, etc, (GARCÍA, 2016, p. 23-25). 
En el caso de la formación docente y, en particular, en la formación investigadora de nuestros profesionales hay dos tipos de competencias referidas en las siguientes líneas:

A) La Alfabetización informacional o competencias informáticas e informativas propiamente dichas de interés ya que, a juicio de Edel, "la usabilidad tanto de las TIC como de la red de redes (www por sus siglas en inglés) estimulan los procesos de pensamiento básico y avanzado en nuestros estudiantes y de qué manera impacta en sus desarrollo de habilidades para la investigación formal" (EDEL NAVARRO, R.; AGUIRRE AGUILAR, G.; BALDERRAMA TRÁPAGA, 2016, p. 211). Incluso en el proceso de formación de investigadores, la interacción que proporciona el campus virtual puede sustituir de algún modo la relación personal entre director y dirigido.

B) La Alfabetización o literacidad académica. Se basa en el desarrollo de la capacidad de los alumnos para saber leer críticamente y saber comunicar los mensajes científicos propio del quehacer docente e investigador. Se comenta por diversos autores la escasa capacidad observada en alumnos universitarios para leer, interpretar y producir textos. Efectivamente, consideramos que la lectura es un instrumento para el desarrollo de las tres culturas: información, conocimiento y comunicación, es decir, leer para comprender la información, generar conocimientos y comunicarlos es también un imprescindible aprendizaje universitario. Se trata de una formación continua encaminada a facilitar la práctica de la información susceptible de transformarse en conocimiento. Es cierto que la práctica de la lectura se contempla como una especie de costumbre inherente a los comienzos de nuestro paso por el mundo. Al niño le preguntamos si ya ha aprendido a leer ya que aprender a leer porque ello nos permite descifrar o descodificar e interpretar pensamientos y mensajes vehiculados en documentos. $Y$ los documentos son imprescindibles porque contienen sensaciones, ideas y pensamientos que los seres humanos sitúan fuera de ellos mismo permitiendo así su consulta y transmisión a lo largo del espacio y del tiempo. Pero ese proceso que llamamos lectura requiere sosiego, tranquilidad y reflexión así como la posibilidad de volver sobre lo leído 
y de pensar sobre lo pensado. Algunos autores afirman que los nuevos modos de educación universitaria, muy basados en las tecnologías, pueden impedir en el alumno el espacio para la creatividad, el pensamiento y el gozo intelectual, lo que la lectura evita al facilitar información con posibilidad de convertirse en ideas. Como se ve, algo contrapuesto a la mera lectura de la imagen y a la observación de las pantallas, proceso marcado por una permanente obsesión por la información novedosa y veloz. $Y$ quedarnos tan solo en el primer elemento nos puede llevar a una sociedad de la ignorancia.

Sirva como ejemplo del peligro que corremos la narración del escritor Juan Manuel de Prada: "Un profesor universitario amigo me confiesa desolado que una amplia mayoría de sus alumnos son por completo incapaces de leer un libro; y que, entre los pocos que afrontan su lectura, sólo un puñado puede comprenderlo... Pero nada ha conturbado tanto a mi amigo como un episodio que le aconteció recientemente: un alumno le solicitó permiso para grabar en vídeo sus clases... el alumno le confesó atribulado que era incapaz de estudiar sus apuntes, porque apenas se ponía a leerlos perdía la concentración. Sólo contemplando el vídeo de sus clases podía llegar a aprender y memorizar las lecciones... Sucedía, sin embargo, que su alumno era incapaz de mantener la atención fija; era incapaz de entender los razonamientos más elementales; era incapaz de seguir el hilo de un relato escrito... en varias conversaciones le reconoció que toda su vida, desde que se levantaba hasta que se acostaba, estaba ligada a los diversos cacharritos y artilugios que le permitían mantenerse on line con amigos y allegados: guasapeando, tuiteando, intercambiando vídeos, hablando por el skype, a veces con varios a la vez, en un intercambio excitante. Inevitablemente, el cerebro de aquel muchacho había acabado por acompasarse a esta vida nerviosa y aturdidora.... Su repudio de la letra impresa era una consecuencia natural de ese aturdimiento; no podía entender un razonamiento mínimamente complejo por la sencilla razón de que su cerebro se exasperaba tratando de hilvanar sus proposiciones, tratando de desentrañar el significado de sus palabras, y buscaba los mensajes inmediatos, netos, ramplones: las consignas, los apóstrofes, los enunciados más sencillos que le permitiesen saltar de inmediato a cualquier otra simpleza que 
irrumpiese, a modo de relámpago fugaz, en su cerebro" (PRADA, 2014).

Por el contrario, las nuevas ideas científicas se obtienen, esencialmente, desde la reflexión y el pensamiento sobre los vestigios aportados por las fuentes mediante la lectura crítica e interpretación idónea de las mismas. Ello tiene lugar desde una disposición subjetiva que comprende las cualidades, la preparación intelectual y el esfuerzo personal del investigador, de una parte, y, de otra, la explotación por el investigador de las ideas contenidas en los trabajos que constituyen el estado de la cuestión, es decir, de las fuentes. Así pues, el ejercicio de interpretación y critica de las fuentes tiene dos finalidades: comprobar su autenticidad y veracidad y aprovechar las ideas, claras o sugeridas, que las mismas contienen. Es condición sine qua non para el correcto ejercicio de interpretación y valoración de las fuentes el desarrollo del espíritu crítico en el investigador, lo que se obtiene mediante un aprendizaje que comporta las siguientes acciones: conocimiento pre- vio, en la medida de lo posible, del contenido del texto que se va a leer, lectura lenta y atenta del mismo y estudio comparado de las argumentaciones de aquellos textos que, teniendo el mismo objeto de investigación, han alcanzado conclusiones radicalmente diferentes y que, con frecuencia, han dado lugar a polémicas científicas

El aprendizaje en la lectura crítica se obtiene a partir del conocimiento previo, en la medida de lo posible, del contenido del texto que se va a leer y ello de modo lento y atento sin dejar pasar palabras cuyo significado no se entienda o frases cuya percepción no esté asegurada. De modo práctico contribuye a lograr tal aprendizaje la lectura comparada de las argumentaciones de aquellos textos que, teniendo el mismo objeto de investigación, han alcanzado conclusiones radicalmente diferentes y que, con frecuencia han dado lugar a polémicas científicas o de otra naturaleza. (LÓPEZ YEPES, 2015)

En efecto, la aplicación de la lectura en el campo de la metodología de la investigación y en la recepción de conocimientos científicos, a partir de los siguientes presupuestos:

1) El hallazgo de las nuevas ideas científicas elaboradas por el investigador, 
como hemos indicado, se basa, de un lado, en el fomento de hábitos como la observación, la tenacidad, la relación entre hechos e ideas, la imaginación, la actitud de duda e incertidumbre permanentes, etc. y, de otro, en el resultado de la reflexión del investigador sobre el contenido de las fuentes. Ello se hace merced al desarrollo de la técnica de la lectura crítica.

2) El acto intelectual en que consiste la lectura crítica descodifica los mensajes ubicados en el documento y permite su interpretación bajo los condicionantes de tiempo, espacio y persona.

3) El investigador no solo interpreta los mensajes de los documentos sino que los integra en su propio texto generando nuevos documentos $y$, por consiguiente, nuevos caminos de lectura crítica e interpretaciones.

4) El aprendizaje de la lectura crítica constituye, en nuestra opinión, un recurso didáctico para lograr en el estudiante la capacidad de obtener ideas propias, acuñar criterios y convertirse en auténtico intelectual.

Para concluir, deseo referirme a una cuestión sobre la que apenas se ha publicado: la formación del asesor o director de investigación. Como es sabido, la formación investigadora se adquiere, primordialmente, a lo largo de su propia actividad. Se aprende a investigar investigando, se suele decir. Y esta actividad, este hacer presenta una doble faz. Un hacer - agere- de carácter inmanente que tiene valor para el investigador en cuanto tarea de aprendizaje por el mero hecho de llevarla a cabo, el actum, y un hacer -facere- de carácter trascendente que proporciona un resultado, el factum, concretado en el texto académico y en el consiguiente título académico.

Pues bien, este aprendizaje se obtiene mediante la ayuda imprescindible de un guía, de un tutor, asesor o director que transmite al investigador, a lo largo de un período de tiempo, hábitos de pensamiento y técnicas que se constituyen como ingredientes de su formación y le facilitan llegar a la meta final. $Y$ todo ello a lo largo de un camino que recorren juntos director y tesista en estrecha relación personal y académica. La relación que se establece entre ambos viene teñida de elementos afectivos y del sentimiento de compartir una aventura intelectual común, lo cual nos lleva a pensar que la tesis es ciencia 
pero es también emoción, nuevo conocimiento pero también pasión, alegría por el descubrimiento pero también tristeza por los numerosos obstáculos que surgen. De ahí que la dirección o asesoramiento de tesis signifique una tarea poliédrica de matices y contenidos y de que la elección de un buen director tenga una trascendencia incalculable habida cuenta de que dicha tarea reúne una serie de hábitos buenos y que, de lo contrario, el tesista puede malformarse y transmitir los efectos nocivos a sus futuros asesorados. Al fin, cabe pensar que el prestigio de la institución científica en que se gesten las tesis dependerá de los factores aludidos, lo que también habrá de repercutir en beneficio de la sociedad.

Todas estas reflexiones las expongo en una obra reciente publicada el año pasado en México ${ }^{3}$. La misma constituye un homenaje a algunos de mis maestros, ilustres catedráticos de universidades españolas que abrieron nuevas vías de investigación y que, con cuyo ejemplo, me impregnaron del amor a la docencia y a la investigación en una institución plena de vida renovada y madre nutricia, a la que todos con orgullo llamamos Universidad.

\section{CONSIDERACIONES FINALES}

1) La Ciencia de la Información/Bibliotecologia/Documentación es una ciencia social, informativo-comunicativa que tiene como objeto de estudio un proceso informativo consistente en la retención, recuperación y transformación de mensajes producidos en procesos informativos anteriores y cuyos mensajes se comunican transformados como fuentes de información para obtener nuevo conocimiento o para la acertada toma de decisiones. Dichos mensajes constituyen la información documental.

2) Las Ciencias de la Información agrupan todas las disciplinas que tienen como objeto de estudio un proceso informativo-documental. Son, pues, la Ciencia de la Información citada en el punto anterior, la Archivística y la Museología.

3 J. LÓPEZ YEPES, La dirección/asesoría de tesis, op. cit. en nota 9.

Inf. Inf., Londrina, v. 22, n. 2, p. 320 - 339, maio/ago., 2017. http:www.uel.br/revistas/informacao/ 
3) La evaluación de la ciencia a base de datos cuantitativo tan solo es, de por si una actividad incompleta, aunque dote a la misma de visibilidad científica. Debe ser completada con indicadores de calidad como nuestra propuesta de análisis cualitativo de citas. Por lo demás, los actuales indicadores alternativos o altmétricos conceden tan solo a día de hoy visibilidad social a la ciencia y a sus protagonistas.

4) El profesional de la información dota de valor añadido a los mensajes discriminándolos en cuanto a su calidad y veracidad $\mathrm{y}$, entre sus competencias, destaca el ejercicio del aprendizaje de la lectura crítica y su incorporación al ámbito de la investigación mediante la obtención de grado de doctor y su incorporación a los equipos de investigación.

\section{REFERÊNCIAS}

AZORÍN. España, $2^{\mathrm{a}}$ ed. Madrid: Espasa-Calpe, 1959.

BORREGO, A. Altmétricas para la evaluación de la investigación y el análisis de necesidades de la investigación. El profesional de la investigación, v. 23, n.4, 2014.

CODINA BONILLA, L. La gran disrupción: la web + los dispositivos móviles y lo que podemos hacer. Informes ThinkEPI sobre Documentación y Comunicación. Barcelona, v.1, s/n, p. 5-7, 2015.

EDEL NAVARRO, R.; AGUIRRE AGUILAR, G.; BALDERRAMA TRÁPAGA, J.A. La contribución didáctica de las TIC en el desarrollo de competencias para la investigación. Ideales, v.1, n.1, p. 209-227, 2016. Disponível em: http://revistas.ut.edu.co/index.php/ldeales/article/view/1080.

GARCíA, J. Las competencias del siglo 21: Un modelo de acercamiento desde la educación. In: GARCÍA, J; LEÓN, T; OROZCO, E. Desarrollo de competencias para el siglo XXI. Miami: Humboldt International University, 2016, p. 15-45.

LÓPEZ YEPES, J. La lectura crítica como recurso didáctico. Modelos y métodos. México DF: Universidad Panamericana, 2015a.

LÓPEZ YEPES, J. La dirección/asesoría de tesis. Materiales para una manual de buenas prácticas. México DF: Universidad Panamericana, 2015b. 
LÓPEZ YEPES, J. La ciencia de la información documental. El documento, la disciplina y el profesional de la información en la era digital. México, D.F.: Universidad Panamericana, 2015.

PRADA, J. M. "Una vida nerviosa". XI Semanal, 7 de febrero de 2014.

TORRES-SALINAS, D.; CABEZAS-CLAVIJO, A. Y.; JIMÉNEZ-CONTRERAS, E. Nuevos indicadores para la comunicación científica en la Web 2.0. Comunicar, v. 21, n. 41, out. 2013.

TORRES-SALINAS, D.; MILANÉS-GUISADO, Y. Presencia en redes sociales y altmétricas de los principales autores de la revista El profesional de la información. El profesional de la información, v. 23, n.4, jul/ago. 2014.

\title{
Title
}

Emerging questions in the Information Science domain

\begin{abstract}
Introduction: the present article reflects on the emerging themes of the Information Science area. It presents some evolving concepts related to the area and discusses aspects around the researches in progress, as well as evaluates the role of information professionals in the scope of scientific research. Objective: To discuss what is Information Science. To understand how research is being evaluated in this scientific field. To study how the information professionals are currently seen and what are the desired competences for these professionals. Methodology: Bibliographic research. Results: The results indicate that information science is a social, informative and communicative science that has as object of study an informational process that consists of retention, retrieval and transformation of information to generate new knowledge.In the scientific reserach context, information Science brings together several áreas of acience that have as objetc of study the informative-documentary process, being the majority of the research of a qualitative nature. Among the various competencies of the information professional, the study emphasizes the reading learning exercise, since this professional acts directly in several areas of knowledge. Conclusions: the study focused on the Information Science area in three aspects: what is the Information Science, what are the most developed researches in the field today and what are the skills of the information professionals. The Information science is a growing area and needs a permanent debate, mainly investigations that cover these three aspects mentioned, since they complement each other.
\end{abstract}

Keywords: Information Science. Information Process. Information Professional Information Professional Competencies.

\section{Título}

Algumas questões emergentes no domínio da Ciência da Informação

Inf. Inf., Londrina, v. 22, n. 2, p. 320 - 339, maio/ago., 2017.

http:www.uel.br/revistas/informacao/ 


\section{Resumo}

Introdução: o presente artigo reflete sobre os temas emergentes da área da Ciência da Informação $(\mathrm{Cl})$. Apresenta alguns conceitos em evolução relacionados à área e discute aspectos em torno das pesquisas em andamento, bem como avalia o papel dos profissionais da informação no âmbito da pesquisa científica. Objetivo: Discutir sobre o que é a $\mathrm{Cl}$. Entender como estão sendo avaliadas as pesquisas neste campo científico. Estudar como são vistos atualmente os profissionais da informação e quais são as competências desejadas para estes profissionais. Metodologia: Pesquisa bibliográfica. Resultados: Os resultados indicam que a $\mathrm{Cl}$ é uma ciência social, informativa/comunicativa que tem como o objeto de estudo um processo informacional que consiste em retenção, recuperação e transformação da informação para geração de novos conhecimentos. No que tange as pesquisas científicas, a $\mathrm{Cl}$ agrupa diversas áreas da ciência que tem como objeto de estudo o processo informativo-documental, sendo a maior parte das pesquisas de natureza qualitativa. Dentre as diversas competências do profissional da informação o estudo enfatiza o exercício de aprendizagem da leitura, uma vez que este profissional atua diretamente em diversas áreas do conhecimento. Conclusões: o estudo focou a área da $\mathrm{Cl}$ em três aspectos, sendo eles: o que é a $\mathrm{Cl}$, quais são as pesquisas mais desenvolvidas no campo atualmente e quais as habilidades dos profissionais da informação. $\mathrm{A} \mathrm{Cl}$ é uma área em crescimento e necessita de um debate permanente, principalmente investigações que abarquem esses os três aspectos mencionados, uma vez que eles se complementam.

Palavras Chave: Ciência da Informação. Processo Informacional. Profissional da Informação. Competências do profissional da Informação.

Recebido: 30.08 .2017

Aceito: 30.09 .2017 